International Journal of Pure and Applied Mathematics

Volume 89 No. 4 2013, 591-617

ISSN: 1311-8080 (printed version); ISSN: 1314-3395 (on-line version)

url: http://www.ijpam.eu

doi: http://dx.doi.org/10.12732/ijpam.v89i4.13

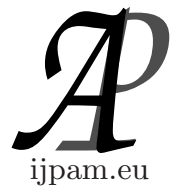

\title{
GLOBAL SYNCHRONIZATION OF HYBRID COUPLED NEURAL NETWORKS WITH INTERVAL TIME-VARYING AND UNBOUNDED DISTRIBUTED DELAYS VIA SAMPLED-DATA FEEDBACK CONTROL
}

\author{
N. Yotha ${ }^{1}$, T. Botmart ${ }^{2} \S$, T. Mouktonglang ${ }^{3}$ \\ ${ }^{1,3}$ Department of Mathematics \\ Chiangmai University \\ Chiangmai, 50200, THAILAND \\ ${ }^{2}$ Department of Mathematics \\ Srinakharinwirot University \\ Bangkok, 10110, THAILAND
}

\begin{abstract}
In this paper, we shall investigate synchronization of neural networks with interval time-varying and unbounded distributed delay with hybrid coupling, which is composed of constant coupling and interval time-varying delays coupling. The designed controller ensures that the synchronization of hybrid couple delayed neural networks are proposed via feedback control and sampled-data feedback control. Based on the construction of improved Lyapunov-Krasovskii functionals and Kronecker product properties, several sufficient conditions are established to achieve global synchronization based on the design of the outer-coupling matrices, the inner-coupling matrices, and some free matrices representing the relationships between the system matrices. New synchronization criteria are derived in terms of LMIs which can be solved efficiently by standard convex optimization algorithms. Two numerical examples are included to show the effectiveness of the proposed feedback control method and sampled-data feedback control scheme.Text of the abstract.
\end{abstract}

AMS Subject Classification: $47 \mathrm{H} 09,47 \mathrm{H} 10$

Received: October 12, 2013

(C) 2013 Academic Publications, Ltd. url: www.acadpubl.eu

${ }^{\S}$ Correspondence author 
Key Words: synchronization, neural networks, interval time-varying and unbounded distributed delays, hybrid coupling, sampled-data feedback control

\section{Introduction}

In the past decade, synchronization in neural networks (NNs), such as cellular NNs, hopfield NNs and bi-directional associative memory networks, has received a great deal of interest among scientists in a variety of areas, such as signal processing, static image processing, associative memory, content-addressable memory and combinatorial optimization $[1,2,3,4,5]$. In performing a periodicity or stability analysis of a neural network, the conditions to be imposed on the neural network are determined by the characteristics of various activation functions and network parameters. When neural networks are created for problem solving, it is desirable for their activation functions are not too restrictive. As a result, there has been considerable research work on the stability of neural networks with various activation functions and more general conditions $[6,7,8]$. On the other hand, the problem of synchronization is an important property of dynamical systems, which is observed in natural, social, physical, chemical and communication engineering, and has found applications in various fields, such as image processing, chaos generators design and harmonic oscillation generation, etc $[9,10,11]$ and references therein. Since synchronization in an array of linearly coupled dynamical systems was investigated in $[12,13]$, many results on local, global and partial synchronization in various coupled systems have also been obtained in $[14,15,16]$. As a special of coupled system, hybrid coupled neural networks have also been found to exhibit some complicated dynamics and even chaotic behaviors and its synchronization has been investigated by many researchers $[17,18,19,20,21]$. Based on the Lyapunov functional method and Kronecker product properties, several sufficient conditions are established to ensure the global exponential synchronization for a general array model of coupled delayed neural networks with hybrid coupling in $[17,18,19]$. Several sufficient conditions for cluster synchronization have been derived based on Lyapunov stability theory and linear matrix inequality (LMI) technique.

Meanwhile, various control approaches such as state feedback control [22, 23], adaptive control [22] and intermittent control [23], are used for controlling and synchronizing the coupled neural networks, most results are based on state-feedback control and adaptive control since they can be proved simply in theoretical and give well control performance. However, in order to take advan- 
tage of the modern high-speed computers, microelectronics, and communication networks, it is more preferable to use digital controllers instead of analog circuits $[24,25,26]$. By following this idea, in [25], the exponential synchronization sampled-data control problem has been studied for neural networks with time-varying mixed delays. Meanwhile, the sampled-data technology has been applied in many practical engineering. For example, the sampled-data synchronization control problem for a class of general complex networks with time-varying coupling delays has been studied in [27]. To the best of the author's knowledge, however, the sampled-data synchronization control problem has not been considered for the hybrid coupled neural networks yet, which is still an open problem and remains challenging.

The stability analysis of time-delay systems has received increasing attention. Time-delay systems are frequently encountered in various areas such as chemical engineering systems, economics and traffic etc, the stability analysis for time-delay systems has been investigated extensively and various approaches to such problems have been proposed, see $[28,29]$ and the references therein. It is well known that the existences of time delay in a system may cause oscillation, divergence and instability system. For these reasons, the stability problem of time-delayed neural networks has been extensively studied for the sake of theoretical interest as well as application considerations [30,31, 32]. According to the nature of how time delay occurs, there are basically two types of time delays, namely, discrete time delays for which the delays can be either constant or time varying $[5,33,34,35]$ and distributed time delays for which the delays are distributed over certain duration of time $[4,36]$. Since discrete and distributed delays can happen simultaneously in signal transmission, most recently, stability analysis for neural networks with both discrete and distributed delays have received considerable research interests $[32,37,38,39]$ and the references therein. Distributed delays including bounded and unbounded distributed delays in neural networks [40, 42, 43, 44] have recently been studied extensively. Nowadays, there are many results dealing with stability and periodic oscillation for neural networks with unbounded distributed delays; see [42, 43, 44]. Some authors have also considered chaos synchronization phenomena in neural networks with unbounded distributed delays: see [40]. It should be pointed out that the synchronization problem for coupled neural networks with discrete and distributed (either bounded or unbounded) has not received enough research attention, and is still an open problem to be addressed. the model studied in this paper contains constant coupling and interval time-varying delay coupling, we call this model the networked model with hybrid coupling

Motivated by the results of the mentioned discussions, we shall investigate 
synchronization of neural networks with interval time-varying and unbounded distributed delay and hybrid coupling. The model studied in this paper contains constant coupling and interval time-varying delay coupling, we call this model the networked model with hybrid coupling. The designed controller ensures that the synchronization of hybrid couple delayed neural networks are proposed via either feedback control or sampled-data feedback control. Based on the construction of improved Lyapunov-Krasovskii functionals and Kronecker product properties, several sufficient conditions are established to achieve global synchronization. New synchronization criteria are derived in terms of LMIs which can be solved efficiently by standard convex optimization algorithms. Numerical examples are given to illustrate the effectiveness and less conservativeness of our theoretical results. To the best of our knowledge, our results are among the first on investigation of global synchronization of hybrid coupled neural networks with interval time-varying and unbounded distributed delays.

The rest of this paper is organized as follows. In Section 2: we give notations, mathematical model of neural networks with interval and unbounded distributed time-varying delays and hybrid coupling delay, assumption, definition and lemma for using in the proof of the main results. Delay-dependent sufficient conditions to guarantee the couple system to be asymptotically synchronized are presented in Section 3. Numerical examples illustrated the obtained results are given in Section 4. The paper ends with conclusions in Section 5 and cited reference.

Notation $R^{n}$ is the $n$-dimensional Euclidean space; $R^{m \times n}$ denotes the set of $m \times n$ real matrices. $I_{n}$ represents the $n$-dimensional identity matrix. The notation $X \geq 0$ (respectively, $X>0$ ) means that $X$ is positive semi-definite (respectively, positive definite). The notation $A \otimes B$ stands for the Kronecker product of matrices $A$ and $B ; \operatorname{diag}(.$.$) denotes a b lock diagonal matrix;$ $\left[\begin{array}{cc}X & Y \\ * & Z\end{array}\right]$ stands for $\left[\begin{array}{cc}X & Y \\ Y^{T} & Z\end{array}\right]$. Matrix dimensions, if not explicitly stated, are assumed to be compatible for algebraic operations.

\section{Preliminaries}

Consider the coupled dynamical system consisting of $N$ linearly and diffusively coupled recurrent neural networks with interval time-varying and unbounded distributed delays can be described by:

$$
\dot{y}_{i}(t)=-C y_{i}(t)+A f\left(y_{i}(t)\right)+B g\left(y_{i}(t-\tau(t))\right)
$$




$$
\begin{aligned}
& +D \int_{-\infty}^{t} k(t-s) h\left(y_{i}(s)\right) d s+E U_{i}(t)+\sum_{j=1}^{N} g_{i j}^{1} \Gamma_{1} y_{j}(t) \\
& +\sum_{j=1}^{N} g_{i j}^{2} \Gamma_{2} y_{j}(t-\tau(t)), \quad i=1,2, \ldots, N
\end{aligned}
$$

where $y_{i}(t)=\left(y_{i 1}(t), y_{i 2}(t), \ldots, y_{i n}(t)\right)^{T} \in R^{n}$ is the state vector of the $i-t h$ network at time $t . C=\operatorname{diag}\left(c_{1}, c_{2}, \ldots, c_{n}\right)>0$ denotes the rate with which the cell $i$ resets its potential to the resting state when being isolated from other cells and inputs, $A, B, D$ and $E$ represent the connection weight matrix, $G^{q}=g_{i j}^{q} \in R^{n \times n},(q=1,2)$ represent the outer-coupling matrix of the network, $\Gamma_{1}, \Gamma_{2} \in R^{n \times n}$ represent a constant inner-coupling matrix, $f\left(y_{i}(t)\right)=$ $\left[f_{1}\left(y_{i 1}(t)\right), \ldots, f_{n}\left(y_{i n}(t)\right)\right]^{T}, g\left(y_{i}(t-\tau(t))\right)=\left[g_{1}\left(y_{i 1}(t-\tau(t))\right), \ldots, g_{n}\left(y_{i n}(t-\right.\right.$ $\tau(t)))]^{T}, h\left(y_{i}(s)\right)=\left[h_{1}\left(y_{i 1}(s)\right), \ldots, h_{n}\left(y_{i n}(s)\right)\right]^{T}$ represent the activation function vectors, $U_{i}(t) \in R^{n}$ represent the control input vector.

The time delays $\tau(t)$ denoted the interval time-varying delay. They are assumed to satisfy the following conditions

$$
0 \leq \tau_{1} \leq \tau(t) \leq \tau_{2}, \dot{\tau}(t) \leq \mu<\infty, \eta=\tau_{2}-\tau_{1},
$$

and $k():.[0,+\infty) \longrightarrow[0,+\infty)$ is the delay kernal.

We denote the synchronization error by $x_{i}(t)=y_{i}(t)-z(t)$ where $z(t) \in R^{n}$ is the state trajectory of the unforced isolate node. Then, the error dynamics of network (1) can be obtained as follows:

$$
\begin{aligned}
\dot{x}_{i}(t)= & -C x_{i}(t)+A f\left(x_{i}(t)\right)+B g\left(x_{i}(t-\tau(t))\right) \\
& +D \int_{-\infty}^{t} k(t-s) h\left(x_{i}(s)\right) d s+E U_{i}(t)+\sum_{j=1}^{N} g_{i j}^{1} \Gamma_{1} x_{j}(t) \\
& +\sum_{j=1}^{N} g_{i j}^{2} \Gamma_{2} x_{j}(t-\tau(t)), \quad i=1,2, \ldots, N,
\end{aligned}
$$

In this paper, we design the feedback controller $U_{i}(t)$ satisfy either $\left(\mathcal{U}_{1}\right)$ or $\left(\mathcal{U}_{1}\right)$ :

$$
\begin{array}{lll}
\left(\mathcal{U}_{1}\right): & U_{i}(t)=K x_{i}(t) & t \geq 0, \\
\left(\mathcal{U}_{2}\right): & U_{i}(t)=K x_{i}\left(t_{k}\right), & t_{k} \leq t<t_{k+1},
\end{array}
$$

where $K \in R^{n \times n}$ is the feedback controller gain matrix to be determined, $t_{k}$ denotes the sampling instant and satisfies

$$
0=t_{0}<t_{1}<\cdots<t_{k}<\cdots<\lim _{k \rightarrow+\infty} t_{k}=+\infty .
$$


Moreover, the sampling period under consideration is assumed to be bounded by a known constant $h$, that is $t_{k+1}-t_{k} \leq h$ for $k \geq 0$.

Clearly, it is difficult to analyze the synchronization of neural networks because of the discrete term $x\left(t_{k}\right)$. Define a sawtooth function as follows:

$$
d(t)=t-t_{k}, t_{k} \leq t<t_{k+1}
$$

It is easily seen that

$$
0 \leq d(t)<h
$$

and the control input of node $i$ can be rewritten as $K x_{i}\left(t_{k}\right)=K x_{i}(t-d(t))$.

Remark 1. In the array of coupled neural networks (2), we have considered the unbounded distributed delay $\int_{-\infty}^{t} k(t-s) h\left(x_{i}(s)\right) d s$. Note that the time-delay $s$ can vary from $-\infty$ to $t$ in a distributed way, hence would have a great impact on the stability of the overall coupled system. There are important practical applications for systems with unbounded distributed delays, see, e.g. $[40,32]$. It is worth mentioning that finite distributed delays, which are another type of distributed delays, have been intensively investigated in [25].

With the Kronecker product, we can reformulate the system (2) as follows:

$$
\begin{aligned}
\frac{d x(t)}{d t}= & -\left(I_{N} \otimes C\right) x(t)+\left(I_{N} \otimes A\right) f(x(t))+\left(I_{N} \otimes B\right) \\
& \times g(x(t-\tau(t)))+\left(I_{N} \otimes D\right) \int_{-\infty}^{t} k(t-s) h(x(s)) d s \\
& +\left(I_{N} \otimes E\right) U(t)+\left(G^{1} \otimes \Gamma_{1}\right) x(t)+\left(G^{2} \otimes \Gamma_{2}\right) x(t-\tau(t)),
\end{aligned}
$$

where

$$
\begin{aligned}
x^{T}(t) & =\left[x_{1}^{T}(t), \ldots, x_{N}^{T}(t)\right] \\
f^{T}(x(t)) & =\left[f^{T}\left(x_{1}(t)\right), \ldots, f^{T}\left(x_{N}(t)\right)\right] \\
g^{T}(x(t-\tau(t))) & =\left[g^{T}\left(x_{1}(t-\tau(t))\right), \ldots, g^{T}\left(x_{N}(t-\tau(t))\right)\right], \\
h^{T}(x(s)) & =\left[h^{T}\left(x_{1}(s)\right), \ldots, h^{T}\left(x_{N}(s)\right)\right] \\
U^{T}(t) & =\left[U_{1}^{T}(t), \ldots, U_{N}^{T}(t)\right]^{T} \\
I^{T}(t) & =\left[I_{1}^{T}(t), \ldots, I_{N}^{T}(t)\right]^{T}
\end{aligned}
$$

and its identical Kronecker product from are

$$
\left(\mathcal{U}_{1}\right): U(t)=\left(I_{N} \otimes K\right) x(t)
$$




$$
\left(\mathcal{U}_{2}\right): \quad U(t)=\left(I_{N} \otimes K\right) x(t-d(t)) .
$$

Throughout this paper, we make the following assumptions:

Assumption 1. The outer-coupling configuration matrices of the complex networks satisfy:

$$
g_{i j}^{q}=g_{j i}^{q} \geq 0, \quad g_{i i}^{q}=-\sum_{j=1, j \neq i}^{N} g_{i j}^{q} .
$$

where $i, j=1,2, \ldots, N, \quad i \neq j$ and $q=1,2$.

Assumption 2. For any constants $l_{r}^{-}, l_{r}^{+}, \sigma_{r}^{-}, \sigma_{r}^{+}, v_{r}^{-}, v_{r}^{+}$, the function satisfy:

$$
\begin{gathered}
l_{r}^{-} \leq \frac{f_{r}\left(s_{1}\right)-f_{r}\left(s_{2}\right)}{s_{1}-s_{2}} \leq l_{r}^{+}, \\
\sigma_{r}^{-} \leq \frac{g_{r}\left(s_{1}\right)-g_{r}\left(s_{2}\right)}{s_{1}-s_{2}} \leq \sigma_{r}^{+}, \\
v_{r}^{-} \leq \frac{h_{r}\left(s_{1}\right)-h_{r}\left(s_{2}\right)}{s_{1}-s_{2}} \leq v_{r}^{+},
\end{gathered}
$$

we denote

$$
\begin{aligned}
& F_{1}=\operatorname{diag}\left(l_{1}^{+} l_{1}^{-}, \cdots, l_{n}^{+} l_{n}^{-}\right), \quad F_{2}=\operatorname{diag}\left(\frac{l_{1}^{+}+l_{1}^{-}}{2}, \cdots, \frac{l_{n}^{+}+l_{n}^{-}}{2}\right), \\
& J_{1}=\operatorname{diag}\left(\sigma_{1}^{+} \sigma_{1}^{-}, \cdots, \sigma_{n}^{+} \sigma_{n}^{-}\right), J_{2}=\operatorname{diag}\left(\frac{\sigma_{1}^{+}+\sigma_{1}^{-}}{2}, \cdots, \frac{\sigma_{n}^{+}+\sigma_{n}^{-}}{2}\right), \\
& L_{1}=\operatorname{diag}\left(v_{1}^{+} v_{1}^{-}, \cdots, v_{n}^{+} v_{n}^{-}\right), L_{2}=\operatorname{diag}\left(\frac{v_{1}^{+}+v_{1}^{-}}{2}, \cdots, \frac{v_{n}^{+}+v_{n}^{-}}{2}\right) .
\end{aligned}
$$

Assumption 3. The delay kernel $k():.[0,+\infty) \longrightarrow[0,+\infty)$ is continuous and integrable, and also satisfies:

$$
\int_{0}^{+\infty} k(s) d s<+\infty, \int_{0}^{+\infty} s k(s) d s<+\infty .
$$

We introduce the following technical well-known Lemma, which will be used in the proof of our results.

Definition 1. System (2) or (7) is said to be globally asymptotically synchronized if the following holds:

$$
\lim _{t \rightarrow \infty}\left\|x_{i}(t)-x_{j}(t)\right\|=0, \quad i, j=1,2, \ldots N, \quad i \neq j .
$$


Lemma 1. Let $\otimes$ denote the notation of Kronecker product. Then, the following relationships hold:

$$
\begin{aligned}
& \text { (1) }(\alpha A) \otimes B=A \otimes(\alpha B) \\
& \text { (2) }(A+B) \otimes C=A \otimes C+B \otimes C \\
& \text { (3) }(A \otimes B)(C \otimes D)=(A C) \otimes(B D) .
\end{aligned}
$$

Lemma 2. [20] Let $e=(1,1, \ldots, 1)^{T}, E_{N}=e e^{T}$, and $U=N I_{N}-E_{N}, P \in$ $R^{n \times n}, x=\left(x_{1}^{T}, \ldots, x_{N}^{T}\right)^{T}$, and $y=\left(y_{1}^{T}, \ldots, y_{N}^{T}\right)^{T}$ with $x_{k}, y_{k} \in R^{n},(k=$ $1,2, \ldots, N)$, then

$$
x^{T}(U \otimes P) y=\sum_{1 \leq i<j \leq N}^{N}\left(x_{i}-x_{j}\right)^{T} P\left(y_{i}-y_{j}\right) .
$$

Lemma 3. Let $H_{1}, H_{2}$ be an $n \times n$ any real matrix, $K$ is an $n \times n$ positive definite matrix, $U, x, y$ are defined as Lemma 2. Then, for any vectors $x$ and $y$ with appropriate dimensions, the following matrix inequality holds:

$$
\pm 2 x^{T}\left(U \otimes H_{1} H_{2}\right) y \leq x^{T}\left(U \otimes H_{1} K^{-1} H_{1}^{T}\right) x+y^{T}\left(U \otimes H_{2}^{T} K H_{2}\right) y
$$

Proof. It is well known that $\pm 2 x^{T} H_{1} H_{2} y \leq x^{T} H_{1} K^{-1} H_{1}^{T} x+y^{T} H_{2}^{T} K H_{2} y$ where $x, y \in R^{n}, H_{1}, H_{2}$ are any real matrices with appropriate dimensions and $K \in R^{n \times n}$ is positive definite matric, then we have:

$$
\begin{gathered}
\pm 2 x^{T}\left(U \otimes H_{1} H_{2} \quad\right) y= \pm 2 \sum_{i=1}^{N-1} \sum_{j=i+1}^{N}\left(x_{i}-x_{j}\right)^{T} H_{1} H_{2}\left(y_{i}-y_{j}\right) \\
\leq \quad x^{T}\left(U \otimes H_{1} K^{-1} H_{1}^{T}\right) x+y^{T}\left(U \otimes H_{2}^{T} K H_{2}\right) y .
\end{gathered}
$$

Lemma 4. [28] For any symmetric positive definite matrix $M>0$, scalar $\gamma>0$ and vector function $\omega:[0, \gamma] \rightarrow R^{n}$ such that the integrations concerned are well defined, the following inequality holds:

$$
\left(\int_{0}^{\gamma} \omega(s) d s\right)^{T} M\left(\int_{0}^{\gamma} \omega(s) d s\right) \leq \gamma\left(\int_{0}^{\gamma} \omega^{T}(s) M \omega(s) d s\right) .
$$

Lemma 5. [40] Let $M$ be a positive semi-definite matrix, $\alpha():.(-\infty, a] \rightarrow$ $[0,+\infty)$ be a scalar function and $F():.(-\infty, a] \rightarrow R^{n}$ be a vector function. If the integrations concerned are well defined, the following inequality holds:

$$
\left(\int_{-\infty}^{a} \alpha(s) F(s) d s\right)^{T} M\left(\int_{-\infty}^{a} \alpha(s) F(s) d s\right)
$$




$$
\leq \int_{-\infty}^{a} \alpha(s) d s\left(\int_{-\infty}^{a} \alpha(s) F^{T}(s) M F(s) d s\right) .
$$

Lemma 6. [28] (Schur complement). Given constant symmetric matrices $X, Y, Z$ with appropriate dimensions satisfying $X=X^{T}, Y=Y^{T}>0$. Then $X+Z^{T} Y^{-1} Z<0$ if and only if

$$
\left(\begin{array}{cc}
X & Z^{T} \\
Z & -Y
\end{array}\right)<0 \quad \text { or } \quad\left(\begin{array}{cc}
-Y & Z \\
Z^{T} & X
\end{array}\right)<0 .
$$

\section{Main Results}

In this section, we present a delay-dependent stabilizability analysis conditions for the given interval time-varying and unbounded distributed delays systems (7), and derive easy-to-verify sufficient conditions to guarantee the coupled system to be globally asymptotically synchronized.

Theorem 2. Assume that assumptions 1-3 hold. The system (7) is globally asymptotically synchronized under the controller (3), if there exist positive definite matrices, $Y, P, Q_{1}, Q_{2}, Q_{3}, Q_{4}, R, S_{1}$,

$S_{2}, S_{3}$, any real matrices $T_{1}, T_{2}$, and positive diagonal matrix $\Lambda, \Omega, \Delta$ with appropriate dimensions, such that the following linear matrix inequalities (LMIs) hold for all $1=i<j=N$ :

$$
\begin{aligned}
\Sigma_{1 i j}= & \mathcal{W}_{i j}-\left[\begin{array}{llllllllll}
0 & 0 & 0 & 0 & 0 & 0 & I & 0 & -I & 0
\end{array}\right]^{T} \\
& \times S_{3}\left[\begin{array}{llllllllll}
0 & 0 & 0 & 0 & 0 & 0 & I & 0 & -I & 0
\end{array}\right]<0, \\
\Sigma_{2 i j}= & \mathcal{W}_{i j}-\left[\begin{array}{llllllllll}
0 & 0 & 0 & 0 & 0 & 0 & -I & I & 0 & 0
\end{array}\right]^{T} \\
& \times S_{3}\left[\begin{array}{llllllllll}
0 & 0 & 0 & 0 & 0 & 0 & -I & I & 0 & 0
\end{array}\right]<0, \\
\Sigma_{3}= & {\left[\begin{array}{ccccc}
-0.1\left(S_{1}+S_{2}\right) & 3 Y^{T} & P E & T_{1} E \\
* & -3 P & 0 & 0 \\
* & & * & -P & 0 \\
* & * & * & -P
\end{array}\right]<0, } \\
\Sigma_{4}= & {\left[\begin{array}{cc}
-T_{2} & T_{2} E \\
-P & -P
\end{array}\right]<0, }
\end{aligned}
$$




$$
\begin{aligned}
& \mathcal{W}_{i j}=\left[\begin{array}{cccccc}
W_{1,1} & W_{1,2} & \left(P+T_{1}\right) B & W_{1,4} & \Omega J_{2} & \Delta L_{2} \\
* & W_{2,2} & T_{2} B & T_{2} A & 0 & 0 \\
* & * & -(1-\mu) Q_{4} & 0 & 0 & 0 \\
* & * & * & -\Lambda & 0 & 0 \\
* & * & * & * & Q_{4}-\Omega & 0 \\
* & * & * & * & * & \bar{k} R-\Delta \\
* & * & * & * & * & * \\
* & * & * & * & * & * \\
* & * & * & * & * & * \\
* & * & * & * & * & *
\end{array}\right. \\
& W_{1,7} \quad S_{1} \quad S_{2} \quad W_{1,10} \\
& \begin{array}{llll}
W_{2,7} & 0 & 0 & T_{2} D
\end{array} \\
& \begin{array}{llll}
0 & 0 & 0 & 0
\end{array} \\
& \begin{array}{llll}
0 & 0 & 0 & 0
\end{array} \\
& \begin{array}{llll}
0 & 0 & 0 & 0
\end{array} \\
& \begin{array}{llll}
0 & 0 & 0 & 0
\end{array} \\
& \begin{array}{llll}
W_{7,7} & 0 & 0 & 0
\end{array} \\
& \text { * } W_{8,8} \quad 0 \quad 0 \\
& \text { * } \quad * \quad W_{9,9} \quad 0 \\
& \text { * * * * } \quad-\frac{1}{k} R
\end{aligned}
$$

where $\bar{k}=\int_{0}^{+\infty} k(s) d s$ and

$$
\begin{aligned}
W_{1,1}= & -P C-C^{T} P-N g_{i j}^{1} P \Gamma_{1}-N g_{i j}^{1} \Gamma_{1}^{T} P-T_{1} C-C^{T} T_{1}^{T} \\
& -N g_{i j}^{1} T_{1} \Gamma_{1}-N g_{i j}^{1} \Gamma_{1}^{T} T_{1}^{T}+\sum_{i=1}^{3} Q_{i}-0.9 S_{1}-0.9 S_{2} \\
& -\Lambda F_{1}-\Omega J_{1}-\Delta L_{1}, \\
W_{1,2}= & -T_{1}-C^{T} T_{2}^{T}-N g_{i j}^{1} \Gamma_{1}^{T} T_{2}^{T}, W_{1,4}=\left(P+T_{1}\right) A+\Lambda F_{2}, \\
W_{1,7}= & -N g_{i j}^{2} P \Gamma_{2}-N g_{i j}^{2} T_{1} \Gamma_{2}, \quad W_{1,10}=\left(P+T_{1}\right) D, \\
W_{2,2}= & \tau_{1}^{2} S_{1}+\tau_{2}^{2} S_{2}+\eta^{2} S_{3}-T_{2}, \quad W_{2,7}=-N g_{i j}^{2} T_{2} \Gamma_{2}, \\
W_{7,7}= & -(1-\mu) Q_{1}-2 S_{3}, \\
W_{9,9}= & -Q_{3}-S_{2}-S_{3} .
\end{aligned}
$$

Moreover the memoryless feedback control is $K=-P^{-1} Y$.

Proof. For the system (7), the following lemma provides a change estimation of Lyapunov-Krasovskii functional candidate:

$$
V\left(t, x_{t}\right)=V_{1}\left(t, x_{t}\right)+V_{2}\left(t, x_{t}\right)+V_{3}\left(t, x_{t}\right)+V_{4}\left(t, x_{t}\right)+V_{5}\left(t, x_{t}\right),
$$


where

$$
\begin{aligned}
V_{1}\left(t, x_{t}\right)= & x^{T}(t)(U \otimes P) x(t) \\
V_{2}\left(t, x_{t}\right)= & \int_{t-\tau(t)}^{t} x^{T}(s)\left(U \otimes Q_{1}\right) x(s) d s \\
& +\int_{t-\tau_{1}}^{t} x^{T}(s)\left(U \otimes Q_{2}\right) x(s) d s \\
& +\int_{t-\tau_{2}}^{t} x^{T}(s)\left(U \otimes Q_{3}\right) x(s) d s \\
& +\int_{t-\tau(t)}^{t} g^{T}(x(s))\left(U \otimes Q_{4}\right) g(x(s)) d s, \\
V_{3}\left(t, x_{t}\right)= & \tau_{1} \int_{-\tau_{1}}^{0} \int_{t+s}^{t} \dot{x}^{T}(\theta)\left(U \otimes S_{1}\right) \dot{x}(\theta) d \theta d s \\
& +\tau_{2} \int_{-\tau_{2}}^{0} \int_{t+s}^{t} \dot{x}^{T}(\theta)\left(U \otimes S_{2}\right) \dot{x}(\theta) d \theta d s, \\
V_{4}\left(t, x_{t}\right)= & \eta \int_{-\tau_{2}}^{-\tau_{1}} \int_{t+s}^{t} \dot{x}^{T}(\theta)\left(U \otimes S_{3}\right) \dot{x}(\theta) d \theta d s, \\
V_{5}\left(t, x_{t}\right)= & \int_{0}^{+\infty} k(s) \int_{t-s}^{t} h^{T}(x(\theta))(U \otimes R) h(x(\theta)) d \theta d s .
\end{aligned}
$$

Calculating the time derivative of $V\left(t, x_{t}\right)$ along the trajectories of $(7)$, noting that the fact that if $X$ is a matrix with zero column sums, then $U X=$ $N X$, we obtain:

$$
\begin{aligned}
\dot{V}_{1}\left(x_{t}\right)= & 2 x^{T}(t)(U \otimes P) \dot{x}(t) \\
= & 2 x^{T}(t)(U \otimes P)\left[-\left(I_{N} \otimes C\right) x(t)+\left(I_{N} \otimes A\right) f(x(t))\right. \\
& +\left(I_{N} \otimes B\right) g(x(t-\tau(t)))+\left(I_{N} \otimes D\right) \\
& \times \int_{-\infty}^{t} k(t-s) h(x(s)) d s+\left(I_{N} \otimes E K\right) x(t) \\
& \left.+\left(G^{1} \otimes \Gamma_{1}\right) x(t)+\left(G^{2} \otimes \Gamma_{2}\right) x(t-\tau(t))\right] \\
\dot{V}_{1}\left(x_{t}\right)= & 2 \sum_{i=1}^{N-1} \sum_{j=i+1}^{N}\left(x_{i}(t)-x_{j}(t)\right)^{T}\left[\left(-P C-N g_{i j}^{1} P \Gamma_{1}+P E K\right)\right. \\
& \times\left(x_{i}(t)-x_{j}(t)\right)+P A\left(f\left(x_{i}(t)\right)-f\left(x_{j}(t)\right)\right) \\
& +P B\left(g\left(x_{i}(t-\tau(t))\right)-g\left(x_{j}(t-\tau(t))\right)\right)
\end{aligned}
$$




$$
\begin{aligned}
& +P D \int_{-\infty}^{t} k(t-s)\left(h\left(x_{i}(s)\right)-h\left(x_{j}(s)\right)\right) d s \\
& \left.-N g_{i j}^{2} P \Gamma_{2}\left(x_{i}(t-\tau(t))-x_{j}(t-\tau(t))\right)\right] .
\end{aligned}
$$

From Lemma 3, we have

$$
\begin{aligned}
& -\sum_{i=1}^{N-1} \sum_{j=i+1}^{N} 2\left(x_{i}(t)-x_{j}(t)\right)^{T} P E P^{-1} Y\left(x_{i}(t)-x_{j}(t)\right) \\
& \leq \sum_{i=1}^{N-1} \sum_{j=i+1}^{N}\left(x_{i}(t)-x_{j}(t)\right)^{T}\left[P E P^{-1} E^{T} P^{T}+Y^{T} P^{-1} Y\right] \\
& \times\left(x_{i}(t)-x_{j}(t)\right) . \\
& \dot{V}_{2}\left(x_{t}\right) \leq \sum_{i=1}^{N-1} \sum_{j=i+1}^{N}\left[\left(x_{i}(t)-x_{j}(t)\right)^{T}\left(Q_{1}+Q_{2}+Q_{3}\right)\left(x_{i}(t)-x_{j}(t)\right)\right. \\
& -(1-\mu)\left(x_{i}(t-\tau(t))-x_{j}(t-\tau(t))\right)^{T} Q_{1}\left(x_{i}(t-\tau(t))\right. \\
& \left.-x_{j}(t-\tau(t))\right)-\left(x_{i}\left(t-\tau_{1}\right)-x_{j}\left(t-\tau_{1}\right)\right)^{T} Q_{2}\left(x_{i}\left(t-\tau_{1}\right)\right. \\
& \left.-x_{j}\left(t-\tau_{1}\right)\right)-\left(x_{i}\left(t-\tau_{2}\right)-x_{j}\left(t-\tau_{2}\right)\right)^{T} Q_{3}\left(x_{i}\left(t-\tau_{2}\right)\right. \\
& \left.-x_{j}\left(t-\tau_{2}\right)\right)+\left(g\left(x_{i}(t)\right)-g\left(x_{j}(t)\right)\right)^{T} Q_{4}\left(g\left(x_{i}(t)\right)\right. \\
& \left.-g\left(x_{j}(t)\right)\right)-(1-\mu)\left(g\left(x_{i}(t-\tau(t))\right)-g\left(x_{j}(t-\tau(t))\right)\right)^{T} \\
& \left.\times Q_{4}\left(g\left(x_{i}(t-\tau(t))\right)-g\left(x_{j}(t-\tau(t))\right)\right)\right] . \\
& \dot{V}_{3}\left(x_{t}\right) \leq \sum_{i=1}^{N-1} \sum_{j=i+1}^{N}\left\{\left(\dot{x}_{i}(t)-\dot{x}_{j}(t)\right)^{T}\left(\tau_{1}^{2} S_{1}+\tau_{2}^{2} S_{2}\right)\left(\dot{x}_{i}(t)-\dot{x}_{j}(t)\right)\right. \\
& -\left[\left(x_{i}(t)-x_{j}(t)\right)-\left(x_{i}\left(t-\tau_{1}\right)-x_{j}\left(t-\tau_{1}\right)\right)\right]^{T} S_{1} \\
& \times\left[\left(x_{i}(t)-x_{j}(t)\right)-\left(x_{i}\left(t-\tau_{1}\right)-x_{j}\left(t-\tau_{1}\right)\right)\right] \\
& -\left[\left(x_{i}(t)-x_{j}(t)\right)-\left(x_{i}\left(t-\tau_{2}\right)-x_{j}\left(t-\tau_{2}\right)\right)\right]^{T} S_{2} \\
& \left.\times\left[\left(x_{i}(t)-x_{j}(t)\right)-\left(x_{i}\left(t-\tau_{2}\right)-x_{j}\left(t-\tau_{2}\right)\right)\right]\right\} \text {. } \\
& \dot{V}_{4}\left(x_{t}\right)=\eta^{2} \dot{x}^{T}(t)\left(U \otimes S_{3}\right) \dot{x}(t) \\
& -\eta \int_{t-\tau_{2}}^{t-\tau_{1}} \dot{x}^{T}(s)\left(U \otimes S_{3}\right) \dot{x}(s) d s
\end{aligned}
$$

let $\eta=\left(\tau_{2}-\tau(t)\right)+\left(\tau(t)-\tau_{1}\right)$, we have

$$
-\eta \int_{t-\tau_{2}}^{t-\tau_{1}} \dot{x}^{T}(s)\left(U \otimes S_{3}\right) \dot{x}(s) d s
$$




$$
\begin{gathered}
=-\left(\tau_{2}-\tau(t)\right) \int_{t-\tau_{2}}^{t-\tau(t)} \dot{x}^{T}(s)\left(U \otimes S_{3}\right) \dot{x}(s) d s \\
-\left(\tau(t)-\tau_{1}\right) \int_{t-\tau_{2}}^{t-\tau(t)} \dot{x}^{T}(s)\left(U \otimes S_{3}\right) \dot{x}(s) d s \\
-\left(\tau_{2}-\tau(t)\right) \int_{t-\tau(t)}^{t-\tau_{1}} \dot{x}^{T}(s)\left(U \otimes S_{3}\right) \dot{x}(s) d s \\
-\left(\tau(t)-\tau_{1}\right) \int_{t-\tau(t)}^{t-\tau_{1}} \dot{x}^{T}(s)\left(U \otimes S_{3}\right) \dot{x}(s) d s .
\end{gathered}
$$

Applying Lemma 4, we have

$$
\begin{aligned}
&-\left(\tau_{2}-\tau(t)\right) \int_{t-\tau_{2}}^{t-\tau(t)} \dot{x}^{T}(s)\left(U \otimes S_{3}\right) \dot{x}(s) d s \\
& \leq-\left[x(t-\tau(t))-x\left(t-\tau_{2}\right)\right]^{T}\left(U \otimes S_{3}\right)\left[x(t-\tau(t))-x\left(t-\tau_{2}\right)\right], \\
&-\quad\left(\tau(t)-\tau_{1}\right) \int_{t-\tau(t)}^{t-\tau_{1}} \dot{x}^{T}(s)\left(U \otimes S_{3}\right) \dot{x}(s) d s \\
& \leq-\left[x\left(t-\tau_{1}\right)-x(t-\tau(t))\right]^{T}\left(U \otimes S_{3}\right)\left[x\left(t-\tau_{1}\right)-x(t-\tau(t))\right] .
\end{aligned}
$$

Let $\beta=\frac{\tau_{2}-\tau(t)}{\tau_{2}-\tau_{1}} \leq 1$. Then

$$
\begin{aligned}
- & \left(\tau_{2}-\tau(t)\right) \int_{t-\tau(t)}^{t-\tau_{1}} \dot{x}^{T}(s)\left(U \otimes S_{3}\right) \dot{x}(s) d s \\
& \leq-\beta\left[x\left(t-\tau_{1}\right)-x(t-\tau(t))\right]^{T}\left(U \otimes S_{3}\right)\left[x\left(t-\tau_{1}\right)-x(t-\tau(t))\right] \\
- & \left(\tau(t)-\tau_{1}\right) \int_{t-\tau_{2}}^{t-\tau(t)} \dot{x}^{T}(s)\left(U \otimes S_{3}\right) \dot{x}(s) d s \\
& \leq-(1-\beta)\left[x(t-\tau(t))-x\left(t-\tau_{2}\right)\right]^{T}\left(U \otimes S_{3}\right)[x(t-\tau(t)) \\
& \left.-x\left(t-\tau_{2}\right)\right]
\end{aligned}
$$

Therefore from (17) and (22), we can estimate $\dot{V}_{4}(t)$ as

$$
\begin{aligned}
& \dot{V}_{4}\left(x_{t}\right) \leq \sum_{i=1}^{N-1} \sum_{j=i+1}^{N}\left\{\eta^{2}\left(\dot{x}_{i}(t)-\dot{x}_{j}(t)\right)^{T} S_{3}\left(\dot{x}_{i}(t)-\dot{x}_{j}(t)\right)\right. \\
& \quad-\left[\left(x_{i}(t-\tau(t))-x_{j}(t-\tau(t))\right)-\left(x_{i}\left(t-\tau_{2}\right)\right.\right. \\
& \left.\left.\quad-x_{j}\left(t-\tau_{2}\right)\right)\right]^{T} S_{3}\left[\left(x_{i}(t-\tau(t))-x_{j}(t-\tau(t))\right)\right. \\
& \left.\quad-\left(x_{i}\left(t-\tau_{2}\right)-x_{j}\left(t-\tau_{2}\right)\right)\right]-\left[\left(x_{i}\left(t-\tau_{1}\right)-x_{j}\left(t-\tau_{1}\right)\right)\right.
\end{aligned}
$$




$$
\begin{gathered}
\left.-\left(x_{i}(t-\tau(t))-x_{j}(t-\tau(t))\right)\right]^{T} S_{3}\left[\left(x_{i}\left(t-\tau_{1}\right)-x_{j}\left(t-\tau_{1}\right)\right)\right. \\
\left.-\left(x_{i}(t-\tau(t))-x_{j}(t-\tau(t))\right)\right]-\beta\left[\left(x_{i}\left(t-\tau_{1}\right)-x_{j}\left(t-\tau_{1}\right)\right)\right. \\
\left.-\left(x_{i}(t-\tau(t))-x_{j}(t-\tau(t))\right)\right]^{T} S_{3}\left[\left(x_{i}\left(t-\tau_{1}\right)-x_{j}\left(t-\tau_{1}\right)\right)\right. \\
\left.-\left(x_{i}(t-\tau(t))-x_{j}(t-\tau(t))\right)\right]-(1-\beta)\left[\left(x_{i}(t-\tau(t))\right.\right. \\
\left.\left.-x_{j}(t-\tau(t))\right)-\left(x_{i}\left(t-\tau_{2}\right)-x_{j}\left(t-\tau_{2}\right)\right)\right]^{T} S_{3}\left[\left(x_{i}(t-\tau(t))\right.\right. \\
\left.\left.\left.-x_{j}(t-\tau(t))\right)-\left(x_{i}\left(t-\tau_{2}\right)-x_{j}\left(t-\tau_{2}\right)\right)\right]\right\} . \\
\dot{V}_{5}\left(x_{t}\right)=\bar{k} h^{T}(x(t))(U \otimes R) h(x(t)) \\
\quad-\int_{0}^{+\infty} k(s) h^{T}(x(t))(U \otimes R) h(x(t)) d s \\
=\bar{k} h^{T}(x(t))(U \otimes R) h(x(t)) \\
-\int_{-\infty}^{t} k(t-s) h^{T}(x(s))(U \otimes R) h(x(s)) d s .
\end{gathered}
$$

Applying Lemma 5, we have

$$
\begin{aligned}
\dot{V}_{5}\left(x_{t}\right) \leq & \bar{k} h^{T}(x(t))(U \otimes R) h(x(t)) \\
& -\frac{1}{\int_{-\infty}^{t} k(t-s) d s}\left(\int_{-\infty}^{t} k(t-s) h(x(s)) d s\right)^{T} \\
& \left.\times(U \otimes R)\left(\int_{-\infty}^{t} k(t-s) h\left(x_{i}(s)\right)\right) d s\right) \\
= & \sum_{i=1}^{N-1} \sum_{j=i+1}^{N}\left\{\bar{k}\left[h\left(x_{i}(t)\right)-h\left(x_{j}(t)\right)\right]^{T} R\left[h\left(x_{i}(t)\right)-h\left(x_{j}(t)\right)\right]\right. \\
& -\frac{1}{\bar{k}}\left(\int_{-\infty}^{t} k(t-s)\left[h\left(x_{i}(s)\right)-h\left(x_{j}(s)\right)\right] d s\right)^{T} R \\
& \left.\times\left(\int_{-\infty}^{t} k(t-s)\left[h\left(x_{i}(s)\right)-h\left(x_{j}(s)\right)\right] d s\right)\right\} .
\end{aligned}
$$

By assumption 2, for any diagonal matrix $\Lambda>0, \Omega>0, \Delta>0$ with appropriate dimensions, it follows that:

$$
\begin{aligned}
& 0 \leq\left[\begin{array}{c}
x_{i}(t)-x_{j}(t) \\
f\left(x_{i}(t)\right)-f\left(x_{j}(t)\right)
\end{array}\right]^{T}\left[\begin{array}{cc}
-\Lambda F_{1} & \Lambda F_{2} \\
\Lambda F_{2} & -\Lambda
\end{array}\right]\left[\begin{array}{c}
x_{i}(t)-x_{j}(t) \\
f\left(x_{i}(t)\right)-f\left(x_{j}(t)\right)
\end{array}\right] \\
& +\left[\begin{array}{c}
x_{i}(t)-x_{j}(t) \\
g\left(x_{i}(t)\right)-g\left(x_{j}(t)\right)
\end{array}\right]^{T}\left[\begin{array}{cc}
-\Omega J_{1} & \Omega J_{2} \\
\Omega J_{2} & -\Omega
\end{array}\right]\left[\begin{array}{c}
x_{i}(t)-x_{j}(t) \\
g\left(x_{i}(t)\right)-g\left(x_{j}(t)\right)
\end{array}\right]
\end{aligned}
$$


$+\left[\begin{array}{c}x_{i}(t)-x_{j}(t) \\ h\left(x_{i}(t)\right)-h\left(x_{j}(t)\right)\end{array}\right]^{T}\left[\begin{array}{cc}-\Delta L_{1} & \Delta L_{2} \\ \Delta L_{2} & -\Delta\end{array}\right]\left[\begin{array}{c}x_{i}(t)-x_{j}(t) \\ h\left(x_{i}(t)\right)-h\left(x_{j}(t)\right)\end{array}\right]$

On the other hand, it is easy to see from the formulation of (7) that the following equations also holds for any matrices $T_{1}, T_{2} \in R^{n \times n}$

$$
\begin{aligned}
0 & =\sum_{i=1}^{N-1} \sum_{j=i+1}^{N} 2\left(x_{i}(t)-x_{j}(t)\right)^{T}\left[-T_{1}\left(\dot{x}_{i}(t)-\dot{x}_{j}(t)\right)+\left(-T_{1} C\right.\right. \\
& \left.-N g_{i j}^{1} T_{1} \Gamma_{1}+T_{1} E K\right)\left(x_{i}(t)-x_{j}(t)\right)+T_{1} A\left(f\left(x_{i}(t)\right)\right. \\
& \left.-f\left(x_{j}(t)\right)\right)+T_{1} B\left(g\left(x_{i}(t-\tau(t))\right)-g\left(x_{j}(t-\tau(t))\right)\right) \\
& -N g_{i j}^{2} T_{1} \Gamma_{2}\left(x_{i}(t-\tau(t))-x_{j}(t-\tau(t))\right) \\
& \left.+T_{1} D \int_{-\infty}^{t} k(t-s)\left(h\left(x_{i}(s)\right)-h\left(x_{j}(s)\right)\right) d s\right] \\
& +2\left(\dot{x}_{i}(t)-\dot{x}_{j}(t)\right)^{T}\left[-T_{2}\left(\dot{x}_{i}(t)-\dot{x}_{j}(t)\right)+\left(-T_{2} C-N g_{i j}^{1} T_{2} \Gamma_{1}\right.\right. \\
& \left.+T_{2} E K\right)\left(x_{i}(t)-x_{j}(t)\right)+T_{2} A\left(f\left(x_{i}(t)\right)-f\left(x_{j}(t)\right)\right) \\
& +T_{2} B\left(g\left(x_{i}(t-\tau(t))\right)-g\left(x_{j}(t-\tau(t))\right)\right)-N g_{i j}^{2} T_{2} \Gamma_{2} \\
& \times\left(x_{i}(t-\tau(t))-x_{j}(t-\tau(t))\right) \\
& \left.+T_{2} D \int_{-\infty}^{t} k(t-s)\left(h\left(x_{i}(s)\right)-h\left(x_{j}(s)\right)\right) d s\right] .
\end{aligned}
$$

From Lemma 3, we have

$$
\begin{aligned}
& -\sum_{i=1}^{N-1} \sum_{j=i+1}^{N} 2\left(x_{i}(t)-x_{j}(t)\right)^{T} T_{1} E P^{-1} Y\left(x_{i}(t)-x_{j}(t)\right) \\
& \quad \leq \sum_{i=1}^{N-1} \sum_{j=i+1}^{N}\left(x_{i}(t)-x_{j}(t)\right)^{T}\left[T_{1} E P^{-1} E^{T} T_{1}^{T}+Y^{T} P^{-1} Y\right] \\
& \left.\quad \times\left(x_{i}(t)-x_{j}(t)\right)\right], \\
& -\sum_{i=1}^{N-1} \sum_{j=i+1}^{N} 2\left(\dot{x}_{i}(t)-\dot{x}_{j}(t)\right)^{T} T_{2} E P^{-1} Y\left(x_{i}(t)-x_{j}(t)\right) \\
& \leq \sum_{i=1}^{N-1} \sum_{j=i+1}^{N}\left[\left(\dot{x}_{i}(t)-\dot{x}_{j}(t)\right)^{T} T_{2} E P^{-1} E^{T} T_{2}^{T}\left(\dot{x}_{i}(t)-\dot{x}_{j}(t)\right)\right.
\end{aligned}
$$




$$
\left.+\left(x_{i}(t)-x_{j}(t)\right)^{T} Y^{T} P^{-1} Y\left(x_{i}(t)-x_{j}(t)\right)\right] .
$$

Let $\zeta_{i j}=\left[\left(x_{i}(t)-x_{j}(t)\right)^{T},\left(\dot{x}_{i}(t)-\dot{x}_{j}(t)\right)^{T},\left(g\left(x_{i}(t-\tau(t))\right)\right.\right.$ $\left.-g\left(x_{j}(t-\tau(t))\right)\right)^{T},\left(f\left(x_{i}(t)\right)-f\left(x_{j}(t)\right)\right)^{T},\left(g\left(x_{i}(t)\right)-g\left(x_{j}(t)\right)\right)^{T}$, $\left(h\left(x_{i}(t)\right)-h\left(x_{j}(t)\right)\right)^{T},\left(x_{i}(t-\tau(t))-x_{j}(t-\tau(t))\right)^{T},\left(x_{i}\left(t-\tau_{1}\right)-x_{j}\left(t-\tau_{1}\right)\right)^{T},\left(x_{i}(t-\right.$ $\left.\left.\left.\tau_{2}\right)-x_{j}\left(t-\tau_{2}\right)\right)^{T},\left(\int_{-\infty}^{t} k(t-s)\left(h\left(x_{i}(s)\right)-h\left(x_{j}(s)\right)\right) d s\right)^{T}\right]^{T}$ Hence, according to (13) - (29), we obtain

$$
\begin{aligned}
\dot{V}\left(x_{t}\right) \leq & \sum_{i=1}^{N-1} \sum_{j=i+1}^{N}\left\{\zeta_{i j}^{T}(t)\left[\left(1-\beta_{i}\right) \Sigma_{1 i j}+\beta_{i} \Sigma_{2 i j}\right] \zeta_{i j}(t)\right. \\
& +\left(x_{i}(t)-x_{j}(t)\right)^{T} \Pi_{1}\left(x_{i}(t)-x_{j}(t)\right) \\
& \left.+\left(\dot{x}_{i}(t)-\dot{x}_{j}(t)\right)^{T} \Pi_{2}\left(\dot{x}_{i}(t)-\dot{x}_{j}(t)\right)\right\},
\end{aligned}
$$

where $\Sigma_{1 i j}$ and $\Sigma_{2 i j}$ are defined in (8), (9), respectively, and

$\Pi_{1}=-0.1 S_{1}-0.1 S_{2}+3 Y^{T} P^{-1} Y+P E P^{-1} E^{T} P+T_{1} E P^{-1} E^{T} T_{1}^{T}$, $\Pi_{2}=-T_{2}+T_{2} E P^{-1} E^{T} T_{2}^{T}$.

Since $0 \leq \beta \leq 1,(1-\beta) \Sigma_{1 i j}+\beta \Sigma_{2 i j}$ is a convex combination of $\Sigma_{1 i j}$ and $\Sigma_{2 i j}$. Therefore, $(1-\beta) \Sigma_{1 i j}+\beta \Sigma_{2 i j}<0$ is equivalent to $\Sigma_{1 i j}<0$ and $\Sigma_{2 i j}<0$. Applying Shur complement lemma, the inequalities $\Pi_{1}$ and $\Pi_{2}$ are equivalent to $\Sigma_{3}<0$ and $\Sigma_{4}<0$. Thus, it follows from (8)-(11) and (30) such that

$$
\lim _{t \rightarrow \infty}\left\|x_{i}(t)-x_{j}(t)\right\|=0
$$

According to Definition 1, we can conclude that system (1) is globally asymptotically synchronized. The proof is completed

Theorem 3. Assume that assumptions 1-3 hold. The system (7) is globally asymptotically synchronized under the controller (4), if there exist positive definite matrices, $Y, P, Q_{1}, Q_{2}, Q_{3}, Q_{4}, R, S_{1}$,

$S_{2}, S_{3}, Z_{1}, Z_{2}$, any real matrices $T_{1}, T_{2}$, and positive diagonal matrix $\Lambda, \Omega, \Delta$ with appropriate dimensions, such that the following linear matrix inequalities (LMIs) hold for all $1=i<j=N$ :

$$
\begin{aligned}
& \Phi_{1 i j}=\mathcal{M}_{i j}-\left[\begin{array}{llllllllllll}
0 & 0 & 0 & 0 & 0 & 0 & I & 0 & -I & 0 & 0 & 0
\end{array}\right]^{T} \\
& \times S_{3}\left[\begin{array}{llllllllllll}
0 & 0 & 0 & 0 & 0 & 0 & I & 0 & -I & 0 & 0 & 0
\end{array}\right]<0, \\
& \Phi_{2 i j}=\mathcal{M}_{i j}-\left[\begin{array}{llllllllllll}
0 & 0 & 0 & 0 & 0 & 0 & -I & I & 0 & 0 & 0 & 0
\end{array}\right]^{T} \\
& \times S_{3}\left[\begin{array}{llllllllllll}
0 & 0 & 0 & 0 & 0 & 0 & -I & I & 0 & 0 & 0 & 0
\end{array}\right]<0,
\end{aligned}
$$




$$
\begin{aligned}
\Phi_{3} & =\left[\begin{array}{ccc}
-0.1\left(S_{1}+S_{2}\right) & P E & T_{1} E \\
* & -P & 0 \\
* & * & -P
\end{array}\right]<0, \\
\Phi_{4}= & {\left[\begin{array}{cc}
-T_{2} & T_{2} E \\
* & -P
\end{array}\right]<0, } \\
\Phi_{5}= & {\left[\begin{array}{cc}
-\frac{1}{h} Z_{2} & 3 Y^{T} \\
* & -3 P
\end{array}\right]<0, }
\end{aligned}
$$

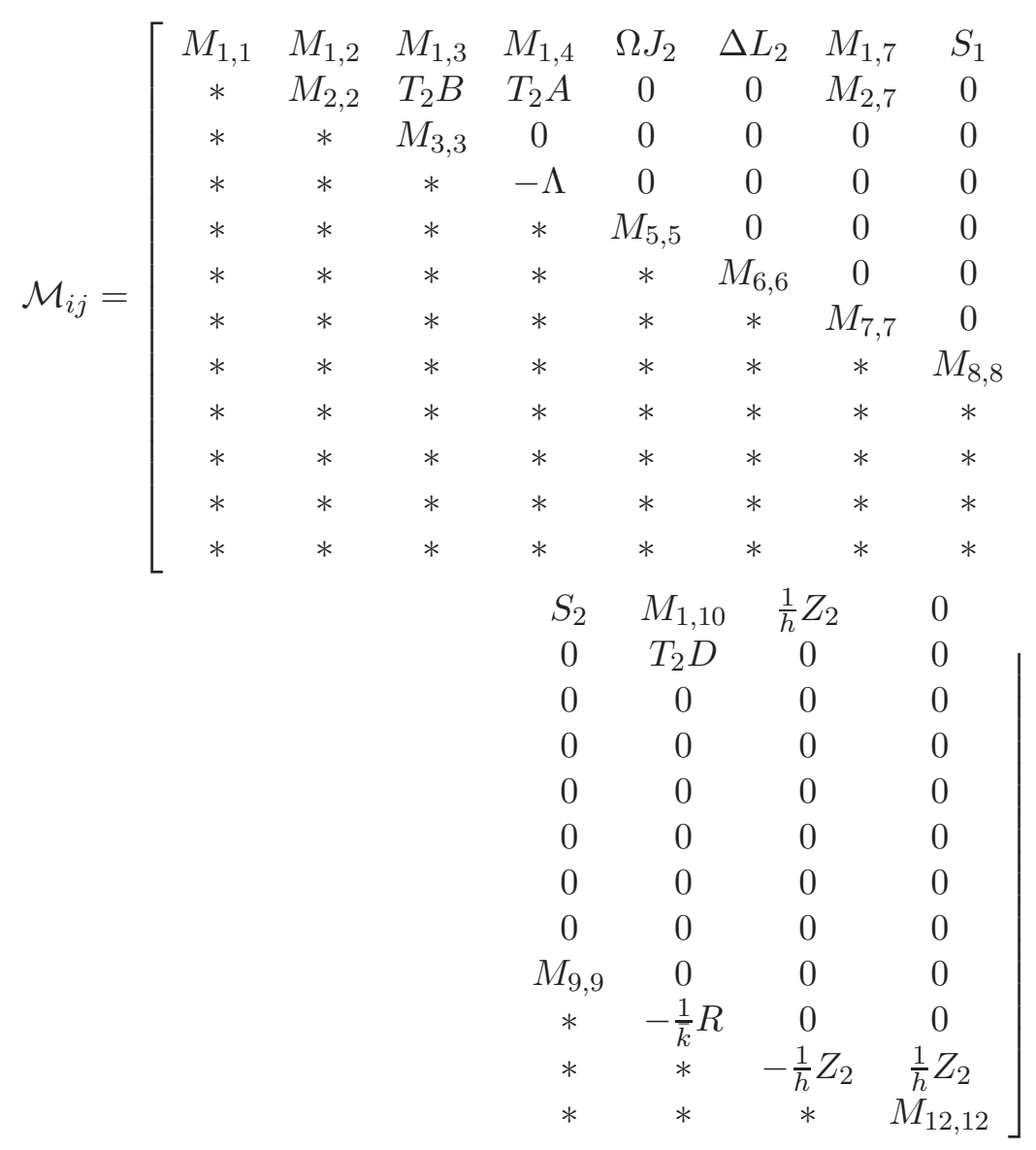

where $\bar{k}=\int_{0}^{+\infty} k(s) d s$ and

$$
\begin{aligned}
M_{1,1}= & -P C-C^{T} P-N g_{i j}^{1} P \Gamma_{1}-N g_{i j}^{1} \Gamma_{1}^{T} P-T_{1} C-C^{T} T_{1}^{T} \\
& -N g_{i j}^{1} T_{1} \Gamma_{1}-N g_{i j} \Gamma_{1}^{T} T_{1}^{T}+\sum_{i=1}^{3} Q_{i}-0.9 S_{1}-0.9 S_{2}
\end{aligned}
$$




$$
\begin{aligned}
& -\Lambda F_{1}-\Omega J_{1}-\Delta L_{1}-\frac{1}{h} Z_{2}+Z_{1}, \\
M_{1,2}= & -T_{1}-C^{T} T_{2}^{T}-N g_{i j}^{1} \Gamma_{1}^{T} T_{2}^{T}, M_{1,3}=\left(P+T_{1}\right) B \\
M_{1,4}= & \left(P+T_{1}\right) A+\Lambda F_{2}, \quad M_{1,7}=-N g_{i j}^{2} P \Gamma_{2}-N g_{i j}^{2} T_{1} \Gamma_{2}, \\
M_{1,10}= & \left(P+T_{1}\right) D, \quad M_{2,2}=\tau_{1}^{2} S_{1}+\tau_{2}^{2} S_{2}+\eta^{2} S_{3}-T_{2}+h Z_{2}, \\
M_{2,7}= & -N g_{i j}^{2} T_{2} \Gamma_{2}, \quad M_{3,3}=-(1-\mu) Q_{4}, \\
M_{5,5}= & Q_{4}-\Omega, \quad M_{6,6}=\bar{k} R-\Delta, \\
M_{7,7}= & -(1-\mu) Q_{1}-2 S_{3}, \quad M_{8,8}=-Q_{2}-S_{1}-S_{3}, \\
M_{9,9}= & -Q_{3}-S_{2}-S_{3}, \quad M_{12,12}=-Z_{1}-\frac{1}{h} Z_{2} .
\end{aligned}
$$

Moreover the memoryless feedback control is $K=-P^{-1} Y$.

Proof. Choose Lypunov-Krasovskii functional as in (12) and adding $V_{6}$, we have

$$
\begin{aligned}
V_{6}\left(t, x_{t}\right)= & \int_{t-h}^{t} x^{T}(s)\left(U \otimes Z_{1}\right) x(s) d s \\
& +\int_{t-h}^{t} \int_{s}^{t} \dot{x}(\theta)\left(U \otimes Z_{2}\right) \dot{x}(\theta) d \theta d s .
\end{aligned}
$$

In addition, by calculating the time derivatives of $V_{6}$, we have

$$
\begin{aligned}
\dot{V}_{6}\left(x_{t}\right) & \leq \sum_{i=1}^{N-1} \sum_{j=i+1}^{N}\left\{\left(x_{i}(t)-x_{j}(t)\right)^{T} Z_{1}\left(x_{i}(t)-x_{j}(t)\right)-\left(x_{i}(t-h)\right.\right. \\
& \left.-x_{j}(t-h)\right)^{T} Z_{1}\left(x_{i}(t-h)-x_{j}(t-h)\right)+h\left(\dot{x}_{i}(t)-\dot{x}_{j}(t)\right)^{T} \\
& \times Z_{2}\left(\dot{x}_{i}(t)-\dot{x}_{j}(t)\right)-\frac{1}{h}\left[\left(x_{i}(t-d(t))-x_{j}(t-d(t))\right)\right. \\
& \left.-\left(x_{i}(t-h)-x_{j}(t-h)\right)\right]^{T} Z_{2}\left[\left(x_{i}(t-d(t))-x_{j}(t-d(t))\right)\right. \\
& \left.-\left(x_{i}(t-h)-x_{j}(t-h)\right)\right]-\frac{1}{h}\left[\left(x_{i}(t)-x_{j}(t)\right)\right. \\
& \left.-\left(x_{i}(t-d(t))-x_{j}(t-d(t))\right)\right]^{T} Z_{2}\left[\left(x_{i}(t)-x_{j}(t)\right)\right. \\
& \left.\left.-\left(x_{i}(t-d(t))-x_{j}(t-d(t))\right)\right]\right\} .
\end{aligned}
$$

Hence, we obtain

$$
\dot{V}\left(x_{t}\right) \leq \sum_{i=1}^{N-1} \sum_{j=i+1}^{N}\left\{\zeta_{i j}^{T}(t)\left[\left(1-\beta_{i}\right) \Phi_{1 i j}+\beta_{i} \Phi_{2 i j}\right] \zeta_{i j}(t)\right.
$$




$$
\begin{aligned}
& +\left(x_{i}(t)-x_{j}(t)\right)^{T} \Upsilon_{1}\left(x_{i}(t)-x_{j}(t)\right)+\left(\dot{x}_{i}(t)-\dot{x}_{j}(t)\right)^{T} \\
& \times \Upsilon_{2}\left(\dot{x}_{i}(t)-\dot{x}_{j}(t)\right)+\left(x_{i}(t-d(t))-x_{j}(t-d(t))\right)^{T} \\
& \left.\times \Upsilon_{3}\left(x_{i}(t-d(t))-x_{j}(t-d(t))\right)\right\},
\end{aligned}
$$

where $\Phi_{1 i j}$ and $\Phi_{2 i j}$ are defined in (31), (32), respectively, and

$$
\begin{aligned}
& \Upsilon_{1}=-0.1 S_{1}-0.1 S_{2}+P E P^{-1} E^{T} P+T_{1} E P^{-1} E^{T} T_{1}^{T}, \\
& \Upsilon_{2}=-T_{2}+T_{2} E P^{-1} E^{T} T_{2}^{T}, \\
& \Upsilon_{3}=-\frac{1}{h} Z_{2}+3 Y^{T} P^{-1} Y .
\end{aligned}
$$

Applying Shur complement lemma, the inequality $\Upsilon_{1}, \Upsilon_{2}$ and $\Upsilon_{3}$ are equivalent to $\Phi_{3}, \Phi_{4}$ and $\Phi_{5}$, respectively, and we may proof the theorem by using a similar argument as in the proof of Theorem 2. Then, that system (1) is globally asymptotically synchronized.

Remark 2. In Theorem 3, the synchronization has been conducted by constructing an appropriate Lyapunov-Krasovskii functionals and Kronecker product properties. It should be mentioned that some newly developed approaches to dealing with interval time-varying delays can be employed to further reduce the conservatism of the stability condition. Research on deriving some less conservative results for the sample-data control problem by using the delay-derivative-dependent approach [45] would be one of our future topics.

\section{Numerical Example}

In this section, we present a simulation example so as to illustrate the usefulness of our main results.

Example 4.1 Consider the system (2) under the controller (3) with the following parameters:

$$
\begin{aligned}
C & =\left[\begin{array}{ll}
5 & 0 \\
0 & 3
\end{array}\right], A=\left[\begin{array}{cc}
1 & -0.2 \\
0.2 & -0.9
\end{array}\right], B=\left[\begin{array}{cc}
0.2 & 0.5 \\
0.2 & -0.1
\end{array}\right], \\
D & =\left[\begin{array}{cc}
-0.1 & 0 \\
0 & -0.1
\end{array}\right], E=\left[\begin{array}{cc}
-0.1 & 0 \\
0.3 & 0.1
\end{array}\right], \Gamma_{1}=\left[\begin{array}{ll}
2 & 0 \\
0 & 2
\end{array}\right], \\
\Gamma_{2} & =\left[\begin{array}{cc}
0.2 & 0 \\
0 & 0.2
\end{array}\right], \tau_{1}=0.2, \tau_{2}=1.0, \mu=0.3, k(s)=e^{-s} .
\end{aligned}
$$


The outer-coupling matrix $G^{q}=g_{i j}^{q} \in R^{n \times n},(q=1,2)$ are defined as:

$g^{1}=\left[\begin{array}{ccccc}-2 & 1 & 0 & 0 & 1 \\ 1 & -3 & 1 & 1 & 0 \\ 0 & 1 & -2 & 1 & 0 \\ 0 & 1 & 1 & -3 & 1 \\ 1 & 0 & 0 & 1 & -2\end{array}\right] g^{2}=\left[\begin{array}{ccccc}-4 & 1 & 1 & 1 & 1 \\ 1 & -4 & 1 & 1 & 1 \\ 1 & 1 & -4 & 1 & 1 \\ 1 & 1 & 1 & -4 & 1 \\ 1 & 1 & 1 & 1 & -4\end{array}\right]$. Take the activation function as follows:

$$
\begin{aligned}
f_{1}\left(x_{i 1}(t)\right) & =0.5\left(\left|x_{i 1}(t)+1\right|-\left|x_{i 1}(t)-1\right|\right), \\
f_{2}\left(x_{i 2}(t)\right) & =0.25\left(\left|x_{i 2}(t)+1\right|-\left|x_{i 2}(t)-1\right|\right), \\
g_{1}\left(x_{i 1}(t)\right) & =\tanh \left(-x_{i 1}(s)\right), \quad g_{2}\left(x_{i 2}(s)\right)=\tanh \left(-1.2 x_{i 2}(s)\right), \\
h_{1}\left(x_{i 1}(s)\right) & =\tanh \left(-x_{i 1}(s)\right), \quad h_{2}\left(x_{i 2}(s)\right)=\tanh \left(-1.2 x_{i 2}(s)\right) .
\end{aligned}
$$

It is easy to see

$$
\begin{aligned}
& F_{1}=\left[\begin{array}{cc}
-1 & 0 \\
0 & -0.25
\end{array}\right], J_{1}=\left[\begin{array}{ll}
0 & 0 \\
0 & 0
\end{array}\right], \quad L_{1}=\left[\begin{array}{ll}
0 & 0 \\
0 & 0
\end{array}\right], \\
& F_{2}=\left[\begin{array}{ll}
0 & 0 \\
0 & 0
\end{array}\right], \quad J_{2}=\left[\begin{array}{cc}
-0.5 & 0 \\
0 & -0.6
\end{array}\right], L_{2}=\left[\begin{array}{cc}
-0.5 & 0 \\
0 & -0.6
\end{array}\right] .
\end{aligned}
$$

By applying the MATLAB LMI Toolbox in Theorem 2, we obtain the the controller gain is

$$
K=\left[\begin{array}{ll}
-0.0369 & -0.0077 \\
-0.0103 & -0.0192
\end{array}\right]
$$

Therefore, it follows from Theorem 2 that the error system (1) under controller gain (39) with given parameters is globally synchronized, which is further verified by the simulation result of the synchronization error given in Figure 1.

Example 4.2 Consider the system (2) under the controller (4) with the following parameters:

$$
\begin{aligned}
C & =\left[\begin{array}{ll}
3 & 0 \\
0 & 2
\end{array}\right], \quad A=\left[\begin{array}{cc}
0.9 & -0.2 \\
0.2 & -0.5
\end{array}\right], B=\left[\begin{array}{cc}
0.5 & 0.5 \\
0.2 & 0.2
\end{array}\right], \\
D & =\left[\begin{array}{cc}
0.1 & 0 \\
0 & 0.1
\end{array}\right], E=\left[\begin{array}{cc}
0.3 & 0 \\
0.1 & 0.3
\end{array}\right], \Gamma_{1}=\left[\begin{array}{cc}
0.2 & 0 \\
0 & 0.2
\end{array}\right], \\
\Gamma_{2} & =\left[\begin{array}{cc}
0.1 & 0 \\
0 & 0.1
\end{array}\right], \tau_{1}=0.2, \tau_{2}=1.0, \mu=0.3, k(s)=e^{-s} .
\end{aligned}
$$




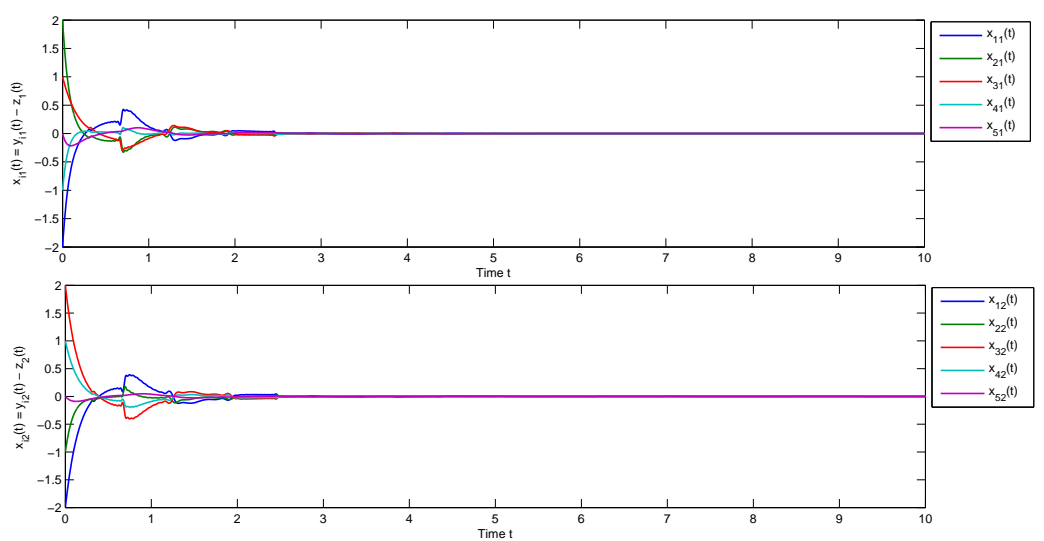

Figure 1: the synchronization errors between states $y_{i}(t), i=1,2, \ldots, 5$ and isolate node $z(t)$

The outer-coupling matrix $G^{q}=g_{i j}^{q} \in R^{n \times n},(q=1,2)$ are defined as:

$g^{1}=\left[\begin{array}{ccccc}-2 & 1 & 0 & 0 & 1 \\ 1 & -3 & 1 & 1 & 0 \\ 0 & 1 & -2 & 1 & 0 \\ 0 & 1 & 1 & -3 & 1 \\ 1 & 0 & 0 & 1 & -2\end{array}\right] g^{2}=\left[\begin{array}{ccccc}-4 & 1 & 1 & 1 & 1 \\ 1 & -4 & 1 & 1 & 1 \\ 1 & 1 & -4 & 1 & 1 \\ 1 & 1 & 1 & -4 & 1 \\ 1 & 1 & 1 & 1 & -4\end{array}\right]$. Take the activation function as follows:

$$
\begin{gathered}
f_{1}\left(x_{i 1}(t)\right)=0.5\left(\left|x_{i 1}(t)+1\right|-\left|x_{i 1}(t)-1\right|\right), \\
f_{2}\left(x_{i 2}(t)\right)=0.25\left(\left|x_{i 2}(t)+1\right|-\left|x_{i 2}(t)-1\right|\right), \\
g_{1}\left(x_{i 1}(t)\right)=0.5\left(\left|x_{i 1}(t)+1\right|-\left|x_{i 1}(t)-1\right|\right), \\
g_{2}\left(x_{i 2}(t)\right)=0.25\left(\left|x_{i 2}(t)+1\right|-\left|x_{i 2}(t)-1\right|\right), \\
h_{1}\left(x_{i 1}(s)\right)=\tanh \left(-x_{i 1}(s)\right), \quad h_{2}\left(x_{i 2}(s)\right)=\tanh \left(-0.8 x_{i 2}(s)\right) .
\end{gathered}
$$

It is easy to see

$$
\begin{aligned}
& F_{1}=\left[\begin{array}{cc}
-1 & 0 \\
0 & -0.25
\end{array}\right], J_{1}=\left[\begin{array}{cc}
-1 & 0 \\
0 & -0.25
\end{array}\right], L_{1}=\left[\begin{array}{ll}
0 & 0 \\
0 & 0
\end{array}\right], \\
& F_{2}=\left[\begin{array}{ll}
0 & 0 \\
0 & 0
\end{array}\right], \quad J_{2}=\left[\begin{array}{ll}
0 & 0 \\
0 & 0
\end{array}\right], \quad L_{2}=\left[\begin{array}{cc}
-0.5 & 0 \\
0 & -0.4
\end{array}\right] .
\end{aligned}
$$


By applying the MATLAB LMI Toolbox in Theorem 3, we obtain the controller gain is

$$
K=\left[\begin{array}{cc}
-0.0866 & 0.0092 \\
0.0128 & -0.1050
\end{array}\right] .
$$

On the other hand, the error system (1) with controller gain (40) is simulated, and the synchronization is observed until the maximum sampling interval $h=0.43$. Therefore, it follows from Theorem 3 with given parameters is globally synchronized, which is further verified by the simulation result of the synchronization error given in Figure 2.
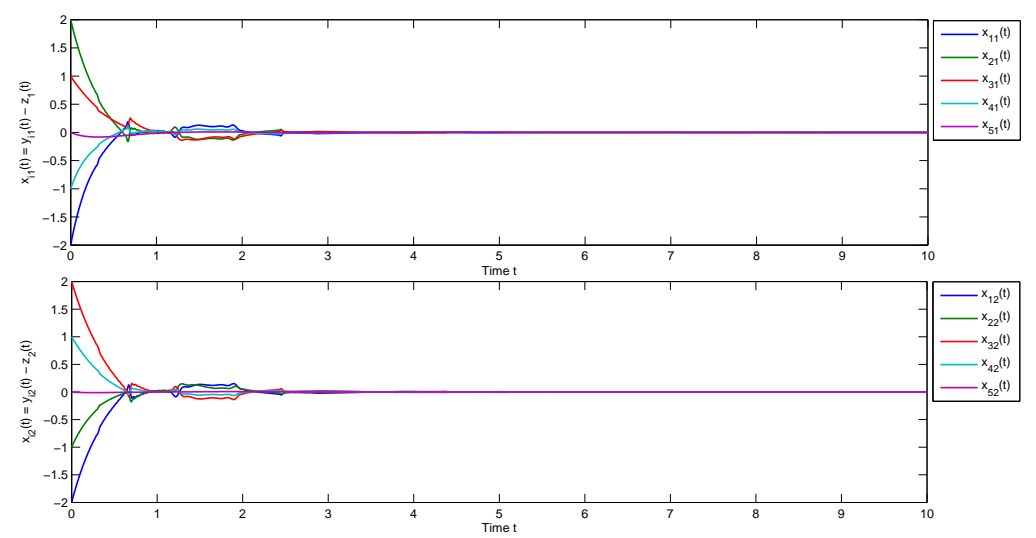

Figure 2: the synchronization errors between states $y_{i}(t), i=1,2, \ldots, 5$ and isolate node $z(t)$

\section{Conclusions}

In this paper, we have been investigated synchronization of neural networks with interval time-varying and unbounded distributed delay and hybrid coupling, which is composed of constant coupling and interval time-varying delay coupling. The designed controller ensures that the synchronization of hybrid couple delayed neural networks are proposed via feedback control and sampled-data feedback control. Based on the construction of improved Lyapunov-Krasovskii functionals and Kronecker product properties, several sufficient conditions are 
established to achieve global synchronization based on the design of the outercoupling matrices, the inner-coupling matrices, and some free matrices representing the relationships between the system matrices. New synchronization criteria are derived in terms of LMIs which can be solved efficiently by standard convex optimization algorithms. Numerical examples are given to illustrate the effectiveness of our theoretical results.

\section{Acknowledgments}

The first author was supported by Chiang Mai University. The second author was supported by the Faculty of Science(283/2555), Srinakharinwirot University.

\section{References}

[1] M. M. Gupta, L. Jin and N. Homma, Static and Dynamic Neural Networks: From Fundamentals to Advanced Theory, New York: Wiley, 2003.

[2] L. M. Pecora and T. L. Carroll, Synchronization in chaotic systems, Phys. Rev. Lett., 64 (1990), 821-824.

[3] A. Cichocki, R. Unbehauen, Neural Networks for Optimization and Signal Processing, Wiley, Hoboken, NJ, 1993.

[4] J. Liang and J. Cao, Global asymptotic stability of bi-directional associative memory networks with distributed delays, Appl. Math. Comput., 152 (2004), 415-424.

[5] O.M. Kwon, S.M. Lee, Ju H. Park and E.J. Cha, New approaches on stability criteria for neural networks with interval time-varying delays, Appl. Math. Comput., 218 (2012), 9953-9964.

[6] M. V. Thuan, Guaranteed cost control of neural networks with various activation functions and mixed time-varying delays in state and control, Differential equations and control processes., 3 (2011), 18-29.

[7] W. Weera and P. Niamsup, Exponential Stabilization of Neutral-Type Neural Networks with Interval Nondifferentiable and Distributed TimeVarying Delays, Abstract and Applied Analysis., (2012), 21 pages. 
[8] V. N. Phat and H. Trinh, Exponential stabilization of neural networks with varous activation functions and mixed time-varying delays, IEEE Trans. Neural Networks., 21 (2010), 1180-1184.

[9] W. Yu, J. Cao and Kun Yuan, Synchronization of switched system and application in communication, Phys. Lett. A, 372 (2008), 4438-4445.

[10] T. -L. Liao and S. -H. Tsai, Adaptive synchronization of chaotic systems and its application to secure communications, Chaos Solitons Fractals., 11 (2000), 1387-1396.

[11] D. Li, Z. Wang, J. Zhou, J. Fang and J. Ni, A note on chaotic synchronization of time-delay secure communication systems, Chaos Solitons Fractals., 38 (2008), 1217-1224.

[12] C. Wu and L. Chua, Synchronization in an array of linearly coupled dynamical systems, IEEE Trans. Circuits Syst. I, 42 (1995), 430-447.

[13] W. Wang and J. Cao, Synchronization in an array of linearly coupled networks with time-varying delay, Physica A, 366 (2006), 197-211.

[14] Y. Wang, H. Zhang, X. Wang and D. Yang, Networked Synchronization Control of Coupled Dynamic Networks With Time-Varying Delay, IEEE, 40 (2010), 1468-1479.

[15] J.Q. Lu, D.W.C. Ho and M. Liu, Globally exponential synchronization in an array of asymmetric coupled neural networks, Phys. Lett. A, 369 (2007) $444-451$.

[16] H. T. Lu and G. R. Chen, Global synchronization in an array of linearly coupled delayed neural networks with an arbitrary coupling matrix, Int. J. Bifurc. Chaos., 16 (2006), 3357-3368.

[17] J. Cao, G. Chen and P. Li, Global synchronization in an array of delayed neural networks with hybrid coupling, IEEE Transactions on Systems, Man and Cybernetics, Part B (Cybernetics), 38(2) (2008), 488-498.

[18] D. Gong, H. Zhang, Z. Wang and B. Huang, Novel synchronization analysis for complex networks with hybrid coupling by handling multitude Kronecker product terms, Neurocomputing, 82 (2012), 14-20.

[19] K. Yuan, Robust synchronization in arrays of coupled networks with delay and mixed coupling, Neurocomputing, 72 (2009), 1026-1031. 
[20] H. Zhang, D. Gong, Z. Wang and D. Ma, Synchronization Criteria for an Array of Neutral-Type Neural Networks with Hybrid Coupling: A Novel Analysis Approach, Neural Process Lett, 35 (2011), 29-45.

[21] J. Cao and L. Li, Cluster synchronization in an array of hybrid coupled neural networks with delay, Neural Networks, 22 (2009), 335-342.

[22] T. Botmart and P. Niamsup, Adaptive control and synchronization perturbed Chuas system, Math. Comput. Simulation, 75 (2007), 37-55.

[23] T. Botmart, P. Niamsup and X. Liu, Synchronization of non-autonomous chaotic systems with time-varying delay via delayed feedback control, Commun. Nonlinea.r Sci. Numer. Simulat., 17 (2012), 1894-1907.

[24] Q. Gan and Y. Liang, Synchronization of chaotic neural networks with time delay in the leakage term and parametric uncertainties based on sampled-data control, Journal of the Franklin Institute, 349 (2012), 19551971.

[25] C. K. Zhang, Y. He and M. Wu, Exponential synchronization of neural networks with time-varying mixed delays and sampled-data, Neurocomputing, 74 (2010), 265-273.

[26] N. Li, J. Hu, J.Hu and L. Li, Exponential state estimation for delayed recurrent neural networks with sampled-data, Nonlinear Dyn, 69 (2012), $555-564$.

[27] N. Li, Y. Zhang, J. Hu and Z. Nie, Synchronization for general complex dynamical networks with sampled-data, Neurocomputing, 74 (2011), 805811.

[28] K. Gu, V.L. Kharitonov and J.Chen, Stability of time-delay system, Boston: Birkhauser, 2003.

[29] H.-Y. Shao, New delay-dependent stability criteria for systems with interval delay, Automatica, 45 (2009), 744-749.

[30] S. Arik, Stability analysis of delayed neural networks, IEEE Trans. Circuits Syst. I., 47 (2000), 1089-1092.

[31] P. Baldi and A. F. Atiya, How delays affect neural dynamics and learning, IEEE Trans. Neural Networks, 5 (1994), 612-621. 
[32] Y. Liu, Z. Wang and X. Liu, Stability criteria for periodic neural networks with discrete and distributed delays, Nonlinear Dyn, 49 (2007), 93-103.

[33] O. M. Kwon and J. H. Park, Exponential stability analysis for uncertain neural networks with interval time-varying delays, Appl. Math. Comput., 212 (2009), 530-541.

[34] J. Tian and X. Zhou, Improved asymptotic stability criteria for neural for networks with interval time-varying delay, Expert Systems with Applications, 37 (2010), 7521-7525.

[35] J. Cao, New results concerning exponential stability and periodic solutions of delayed cellular neural networks, Phys. Lett. A, 307 (2003), 136-147.

[36] J. H. Park, On global stability criterion of neural networks with continuously distributed delays, Chaos Solitons Fractals, 37 (2008), 444-449.

[37] J. H. Park, Further results on asymptotic stability criterion of cellular neural networks with time-varying discrete and distributed delays, Appl. Math. Comput., 182 (2006), 1661-1666.

[38] J. H. Park and H. J. Cho, A delay-dependent asymptotic stability criterion of cellular neural networks with time-varying discrete and distributed delays, Chaos Solitons Fractals, 33 (2007), 436-442.

[39] J. H. Park, An analysis of global robust stability of uncertain cellular neural networks with discrete and distributed delays, Chaos Solitons Fractals, 32 (2007), 800-807.

[40] Y. Liu, Z. Wang and X. Liu, On synchronization of coupled neural networks with discrete and unbounded distributed delays, Int. J. Comput. Math., 85 (2008), 1299-1313.

[41] Q. Song and Z. Zhao, Global dissipativity of neural networks with both variable and unbounded delays, Chaos Solitons Fractals, 25 (2005), 393401.

[42] H. Yang and T. Chu, LMI conditions for stability of neural networks with distributed delays, Chaos Solitons Fractals, 34 (2007), 557-563.

[43] Z. Yi, P.A. Peng, K.S. Leung, Convergence analysis of cellular neural networks with unbounded delay, IEEE Transactions on Circuits and Systems. I, 48 (2001), 680-687. 
[44] J. Zhang, Y. Suda, T. Iwasa, Absolutely exponential stability of a class of neural networks with unbounded delay, Neural Networks, 17 (2004), 391-397.

[45] X. M. Zhang and Q.L. Han Novel delay-derivative-dependent stability criteria using new bounding techniques Int. J. Robust. Nonlinear Control, 23 (2013), 1419-1432. 
